\title{
THE WAVELENGTH VARIATION OF THE DIRECTIONAL SENSITIVITY OF THE STILES $\pi_{1}(\mu)$
}

\author{
M. AlPERn and F. ZWAS \\ Vision Research Laboratory, The University of Michigan, Ann Arbor, Michigan 48109, U.S.A.
}

(Received 19 September 1978; in revised form 30 May 1979)

\begin{abstract}
The variation of the directional sensitivity of Stiles' (1953) $\pi_{1}(\mu)$ mechanism was measured throughout the spectrum on one deuteranope. For wavelengths greater than about $550 \mathrm{~nm}$, the directional properties measured for $\pi_{1}(\mu)$ are nearly identical to those for this deuteranope's $\pi_{3}(\mu)$ mechanism. This supports the hypothesis of Pugh (1976) that $\pi_{1}(\mu)$ is a composite mechanism so that in the yellowred part of the spectrum its sensitivity is determined by long- (and/or medium-) wave sensitive cones. It also obviates the contradiction between the single $\pi_{1}(\mu)$ directional sensitivity result of Stiles (1939) and the suggestion (Enoch and Stiles, 1961) that short-wave sensitive cones are more sensitive to light entering through the edge than through the center of the pupil. This deuteranope's long-wave sensitive cones appear to be most sensitive to backgrounds entering the eye $0.5 \mathrm{~mm}$ nasal, to the center of his pupil while his short-wave sensitive cones appear to be most sensitive to light entering $0.2 \mathrm{~mm}$ temporal to the center of the pupil. Differences in the estimate of losses in the lens below $450 \mathrm{~nm}$ in the spectrum are too large to exclude either self-screening or the Snyder-Pask waveguide theories as applied to this subject's short-wave sensitive cones.
\end{abstract}

\section{INTRODUCTION}

In the previous paper one of us (Zwas, 1979) studied the directional sensitivity of long-wave sensitive cones of deuteranopes, as well as that of the medium-wave sensitive cones of protanopes, as a function of wavelength. This paper evaluates the directional sensitivity of $\pi_{1}(\mu)$, the short-wave sensitive cone mechanism of Stiles (1939).

Estimates of the wavelength dependence of directional sensitivity of short-wave sensitive cones have been made in two ways: (i) Enoch and Stiles (1961) assumed reasonable action spectra of the three kinds of cones for central pupil entry of the light and calculated the changes in these spectra needed to account for the change in color of a monochromatic light as it was moved from the center to the edge of the pupil. (ii) Stiles (1939) measured the change in threshold with change in the point of pupil entry for a monochromatic test for the short-wave sensitive mechanism [later (1953) identified as $\pi_{1}$ ] as a function of test wavelength $\lambda$, the latter being equal to, or smaller than, $500 \mathrm{~nm}$. He found essentially no change in the action spectrum of $\pi_{1}$ with change in the point of pupil entry except at the two lowest wavelengths $410 \mathrm{~nm}$ and $427 \mathrm{~nm}$ for which changes in path length through the lens and macular pigment may well have caused considerable prereceptor distortion. On the other hand, the calculation from matching predicts a precipitous drop in the directional sensitivity of shortwave sensitive cones in the red-green spectral range so that, for example, for sodium yellow light, blue cones are estimated to be more sensitive to light through the pupil's edge than through its center (Enoch and Stiles, 1961).

Unfortunately, thresholds (Stiles, 1939) are not easily determinable for $\pi_{1}$ at test wavelengths longer than $500 \mathrm{~nm}$ - precisely those for which calculation from matching leads to this surprising expectation.
Stiles did, however, determine two-color increment threshold (tvi) curves for one central and one peripheral point of pupil entry of the background field (the test entering the eye always through the pupil center) for a single test-background combination ( $480 \mathrm{~nm}$ test on $580 \mathrm{~nm}$ background). Enoch and Stiles' calculation to the contrary not withstanding, the yellow background entering the eye through the edge of the pupil had to be brighter (not dimer) than that required when the background entered the eye through the pupil center in order to raise the threshold for $\pi_{1}$ by one $\log _{10}$ unit. This means that $\pi_{1}(\mu)$ was less (rather than more) sensitive to backgrounds entering the eye through edge of the pupil compared with its sensitivity to backgrounds entering the eye through the pupil center. In the present paper this last experiment has been repeated on a single subject (a deuteranope) at seven different pupil entry points of the background field not only for a background wavelength of $580 \mathrm{~nm}$, but for 16 other wavelengths in the visible spectrum as well. From these measurements one can obtain for each point of pupil entry of the background the intensity of the latter required to elevate the threshold of $\pi_{1}$ by one $\log _{10}$ unit for every background wavelength. In this way the field sensitivity action spectra (Stiles, 1978) for the various points of pupil entry of the background were determined for the first time on a single foveal cone mechanism.

\section{Apparatus}

\section{METHOD}

A plan view of the optical system is shown in Fig. 1. Three channels are illuminated by the same source, a $150 \mathrm{~W}$ xenon arc with suitable stable power supply. Identical lenses $L_{1}$ imaged the arc and $L_{2}$ collimated the beams in each channel.

Channel 3 was used for the test field. A sectored disc $S D$, in the plane of the arc image, interrupted the light 


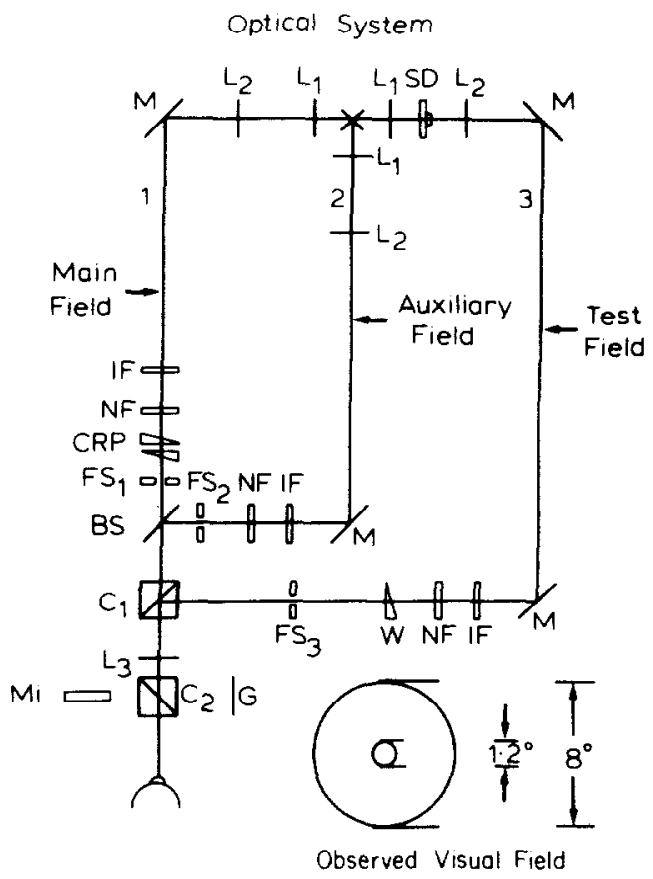

Fig. 1. Optical system. The components of the system are discussed in the text. The insert, lower right, shows the spatial distribution of the test, the main and auxiliary fields.

in this beam exposing a $200 \mathrm{msec}$ flash once each second The field stop $F S_{3}$ limited the size of this field to $1.2^{\circ}$ Narrow band (10 nm half width) Baird Atomic B-1 interference filters $(I F)$ specified the test dominant wavelengths either $450 \mathrm{~nm}$ (whenever the auxiliary background was used) or $480 \mathrm{~nm}$ (when it was not). Inconel neutral filters $N F$ and a calibrated and counterbalanced Wratten No. 96 wedge $\boldsymbol{W}$ which the subject adjusted by hand, attenuated the light to threshold.

Channel 2 contained a circular stop $\left(\mathrm{FS}_{2}\right)$ limiting the field size to $8^{\circ}$ in diameter. This was used as an auxiliary background and contained both neutral and an interference filter in combination to yield $9.642 \mathrm{log}$ quanta $\mathrm{sec}^{-1} \mathrm{deg}^{-2}$ of $600 \mathrm{~nm}$ monochromatic light.

Channel 1 contained a similar stop $F S_{1}$ of the same size as $F S_{2}$, neutral filters, interference filters $(I F)$ the dominant wavelengths of which were varied parametrically, and a pair of counter-rotating (Risley) prisms which moved the point of entry of this field (the main background) across the pupil as a second parameter. $F S_{1}$ was mounted on a slide with two-dimensional micrometer adjustments so that as this beam entered the eye through different pupillary positions the misalignment of its image on the retina due to aberrations in the eye's optics could be corrected.

The three field stops $F S_{1}, F S_{2}$ and $F S_{3}$ were all mounted in the anterior focal plane of the lens $L_{3}(155 \mathrm{~mm} \mathrm{f} / 2$ Ektar) while the eye's entrance pupil and the ruled grid $G$ were in its posterior focal plane. Both of the latter could be viewed by the experimenter through the microscope $M i$ and the mixing cube $C_{2}$ allowing (i) exact alignment of the corneal reflex, (ii) calibration of the pupillary traverse of the background beam, and (iii) monitoring alignment and fixation during the experiment. The circular arc image provided by Channel 3 in the entrance pupil plane was $0.25 \mathrm{~mm}$ in diameter, those by the backgrounds were $1.0 \mathrm{~mm}$.

The relative spectral distribution of the light after attenuation by the optics was measured with each interfer- ence filter in the beam with the aid of calibrated pir-10 silicon photodiode (United-Detector Technology). All filters and wedges were calibrated with a Beckman Acta II spectrophotometer.

\section{Procedure}

The subject's pupil was dilated with one drop of $1 \%$ cyclopentolate hydrochloride about $30 \mathrm{~min}$ before each experimental session and his head fixed by a wax dental bite mounted in a heavy drill press table with three micrometer adjustments. He required no correcting lenses.

The test beam (and the auxiliary field when it was used) entered through the center of the pupil; the background beam traversed along the horizontal meridian vertically set through the pupil center.

\section{A. The $\pi$ mechanisms in deuteranopia (preliminary} experiments)

Increment thresholds were measured in the manner of Stiles (1939) on one deuteranope. There is strong evidence (Rushton, 1965; Alpern and Wake, 1977) that such dichromats have only two cone visual pigments - cyanolabe and erythrolabe--underlying all foveal vision. This introduces a simplification in the analysis but raises an uncertainty whether the experimental conditions employed by Stiles (1939) with normal trichromats isolate comparable mechanisms in this dichromat. Therefore, a series of preliminary experiments measured the increment threshold for a $480 \mathrm{~nm}$ test as a function of background intensity $(\mu)$ with all rays entering the eye through the center of the pupil. (In normals this isolates $\pi_{4}$ and $\pi_{1}$ ).

The absolute threshold for a blue $(480 \mathrm{~nm})$ test for some subjects in these viewing conditions may be determined by rods either because: (i) their foveas contain rods or at least the rod-free area may be smaller than that excited by the 1.2 test, (ii) they may fail to maintain strict foveal fixation or (iii) the rod-free area may not be centered on the fovea. Whatever the cause, this difficulty may be overcome by making absolute thresholds measurements (as well as those against the dimmest backgrounds) between 5 and $7 \mathrm{~min}$ in the dark after a very bright bleach during a time interval in which the rod threshold is very much higher than that of the fully dark-adapted cones. Stiles (1946) was the first both to emphasize these difficuities and to validate the procedure as the most suitable method for obviating them in the determination of the absolute threshold for $\pi_{1}$ under the present viewing conditions. It is also used here.

Figure 2 shows a series of threshold versus intensity (tvi) curves. The one on the extreme left gives results obtained on $510 \mathrm{~nm}$ background; it is properly placed on the abscissa scale. All the other sets of data have been displaced arbitrarily to the right, each $0.75 \log _{10}$ unit further than its left-hand neighbor. Each set is fit with smooth curves defined by Stiles' $\zeta$ function (Wyszecki and Stiles, 1967). For the left-most set, a single branch curve defines all the data, but for backgrounds longer than $510 \mathrm{~nm}$ wavelength, two curves each defining a different $\pi$ mechanism are found. With those backgrounds whenever the test exceeds a value about $1.4 \mathrm{log}$ units above its threshold in the dark a "change in law" occurs as this second $\pi$ mechanism $\left[\pi_{1}(\mu)\right]$ comes into play. Since this subject is a deuteranope the lower template defines $\pi_{5}(\mu)$.

Readers meeting it for the first time may be curious about the application of a single template curve following the displacement rules of Stiles (1939, 1949, 1953, 1959. 1978) to order all our experimental results. A discussion of the two simple assumptions which underly this procedure is beyond the scope of the present work, particularly as a very recent paper by Stiles (1978), to which readers are referred, deals with these matters in detail. Aside from the reasonableness of these assumptions, the justifications for the procedure (and the constraints on its application) are 


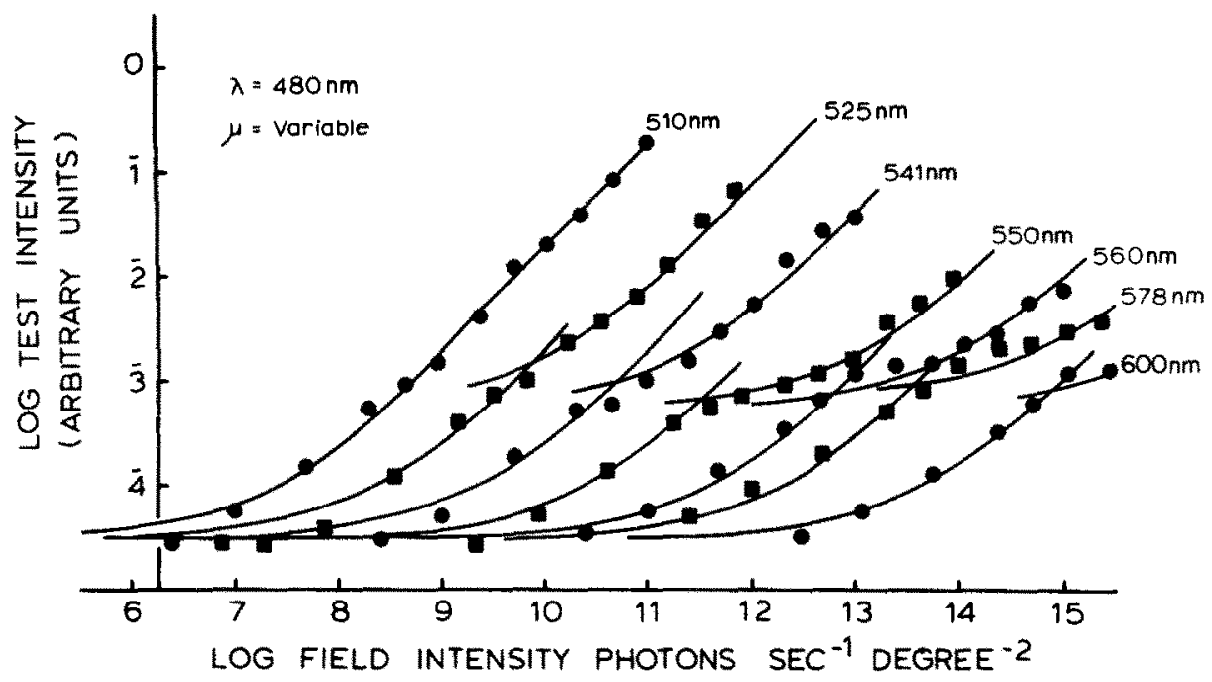

Fig. 2. Increment threshold vs intensity (tvi) curves in which both the main and test fields entered through the center of the pupil. The test field was $480 \mathrm{~nm}$; the background wavelengths were varied parametrically as indicated. The extreme left hand curve $(\mu=510 \mathrm{~nm})$ is properly placed on the abscissa while all the other curves are shifted successively to the right by 0.75 of a log unit. The smooth curves drawn through the points is the standard increment threshold $(\zeta)$ function of Stiles. The lower and upper branches represent $\pi_{5}$ and $\pi_{1}$. respectively (cf. Fig. 4 for action spectra).

largely to be found in the great mass of experimental results (Stiles, 1939, 1949, 1953, 1959, 1967; Pugh, 1976; Cavonius and Estevez, 1975; Estevez and Cavonius, 1977; Sigel, 1976; Pugh and Sigel, 1978; Du Croz and Rushton, 1966; Krauskopf and Mollon, 1971; Mollon and Polden, 1977a, b; Augenstein and Pugh, 1977 to mention only a few of the relevant papers) which are ordered by it and by the remarkable prescience by which, for example, applying it to psychophysical measurements on man, predict the absorption spectra of primate red and green cone pigments nearly 40 years before the fact of their direct measurement by microspectrophotometry (Bowmaker et al., 1978). Familiarity with this literature is implicit to what follows in this paper.

To apply this procedure, decisions had to be reached as to absolute thresholds of $\pi_{1}$ and $\pi_{5}$ as well as the precise amount of lateral shifting of the template required for best fit for each mechanism. Both of these judgments were made by eye in such a way as to minimize deviations from the curve, and are to some extent arbitrary; the former less so than the latter. The precision of the fitting procedure based on these decisions is given for each mechanism at every wavelength and each point of pupil entry in the Appendix. Slightly different judgments in fitting might be made, no doubt, without causing an appreciable loss in this precision. Such changes while they would alter to some slight extent the numbers in the subsequent analysis, would not change the conclusion inferred from them in a substantive way.

In Fig. 2 the tvi curve for a background of $510 \mathrm{~nm}$ (and as Stiles has shown, for shorter background wavelengths) are fit by only a single $\zeta$ template, because $\pi_{1}(\mu)$ no longer determines threshold even at high field intensities. To study this latter mechanism at these background wavelengths, Stiles (1953) added a long wave "auxiliary" background intense enough so that the threshold of the short wavelength test stimulus on it alone was determined by $\pi_{1}$. Figure 3 illustrates this procedure as it applies to the present subject. The circles plot the tvi curve for the violet $(\lambda=450 \mathrm{~nm})$ test on the red auxiliary field $\left(\mu_{a}=600 \mathrm{~nm}\right)$. Under these conditions action spectrum measurements show that at $9.64 \log$ photons $\sec ^{-1} \mathrm{deg}^{-2} \pi_{1}$ threshold is $1.05 \log$ units more sensitive than the next most sensitive foveal mechanism [i.e. $\left.\pi_{5}(\mu)\right]$. The $600 \mathrm{~nm}$ field was fixed at this intensity and the threshold for the same violet test was measured on an additional "main" background (in the illustrated case it is $500 \mathrm{~nm}$ ) superimposed on the $600 \mathrm{~nm}$ "auxiliary" field as the intensity of the $500 \mathrm{~nm}$ field was varied (triangles in Fig. 3). The field sensitivity for the $\pi_{1}(500)$ could then be determined by the intensity of the $500 \mathrm{~nm}$ background required to elevate the threshold for this mechanism by one log unit with the assistance of the $\zeta$ template fit to the triangles in this figure $[9.80 \mathrm{log}$ photons $\left.(500 \mathrm{~nm}) \mathrm{sec}^{-1} \mathrm{deg}^{-2}\right]$.

According to the Stiles assumptions the field sensitivity for $\pi_{1}(\mu)$ determined in the two ways just described (i.e. with, and without, the auxiliary background) should agree. However, Pugh (1976) very recently has found that a mix* ture of a long- and a short-wave background has a "super additive" effect on $\pi_{1}(\mu)$ not predicted by these assumptions, so that a given main background will be mote effective in elevating the threshold of $\pi_{1}$ if superimposed on an auxiliary background than will be the case when it is presented without that background. This finding means that the action spectrum obtained on the auxiliary background will be more sensitive at any given wavelength than expected from Stiles assumptions. We obviated this diff:culty by measuring at a single background (i.e. $\mu=525$ ) the field sensitivity for $\pi_{1}(\mu)$ by both methods. It was found that with the auxiliary field the intensity of the $525 \mathrm{~nm}$ main background required to elevate $\pi_{1}$ threshold one log unit had to be increased by a factor of 2.88 in order to obtain that field intensity measured without the auxiliary field. This directly measured "superadditivity factor" is in qualitative agreement with Pugh's results. Accordingly, in fitting field sensitivity action spectrum for $\pi_{1}(\mu)$ (for example, in Figs 4 and 9) all main background intensities at the criterion, obtained on the auxiliary background were increased by this factor.

With this modification the action spectra obtained in these two sets of preliminary experiments are illustrated by the solid circles (for the short-wave sensitive mechanism) and solid triangles (long-wave sensitive mechanism) in Fig. 4.

The curves in this figure are the field sensitivity action spectra of $\pi_{1}(\mu)$ and $\pi_{5}(\mu)$ of Stiles (Wyszecki and Stiles, 1967, Table 7.6, p. 579). Note that nothing has been arbitrarily shifted. While the agreement is far from perfect (par- 


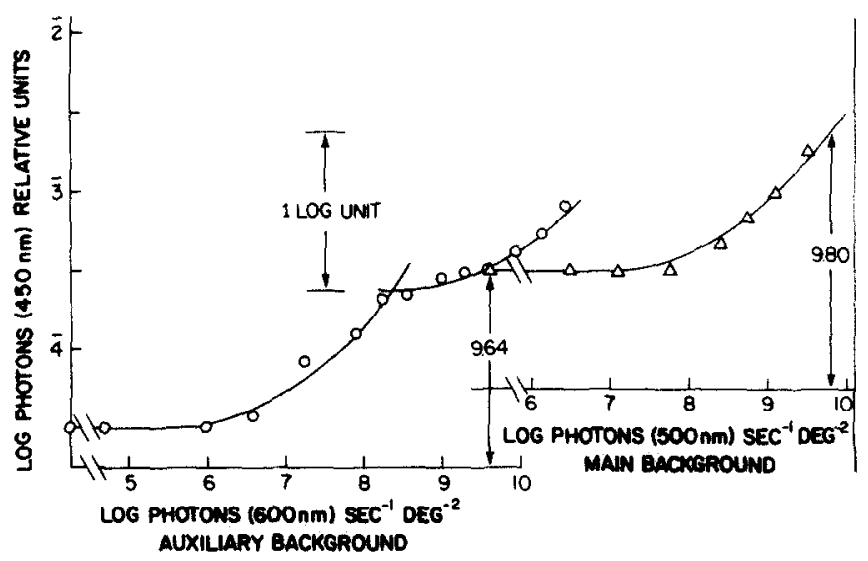

Fig. 3. Illustration of the principle of the auxiliary background. The open circles show the tvi curves for a violet $(\lambda=450 \mathrm{~nm})$ test on an orange $\left(\mu_{a}=600 \mathrm{~nm}\right)$ background; the two smooth curves are the $\zeta$ template fit, below to $\pi_{5}$, above to $\pi_{1}$. At $9.64 \log$ photons this background elevates the threshold for $\pi_{1}$ by $0.125 \log$ unit and the threshold for $\pi_{5}$ is then higher than $\pi_{1}$ by $1.05 \log$ units (estimated by extrapolation along the template fit to the lower branch). The open triangles are results obtained when a main background $(\mu=500 \mathrm{~nm})$ is superimposed on $\mu_{a}$ at this level. $9.80 \log _{10}$ photons $(500 \mathrm{~nm})$ $\mathrm{sec}^{-1} \mathrm{deg}^{-2}$ of the main field elevates $\pi_{1}$ by a factor of 10 . The smooth curve through the triangles is also the $\zeta$ function.

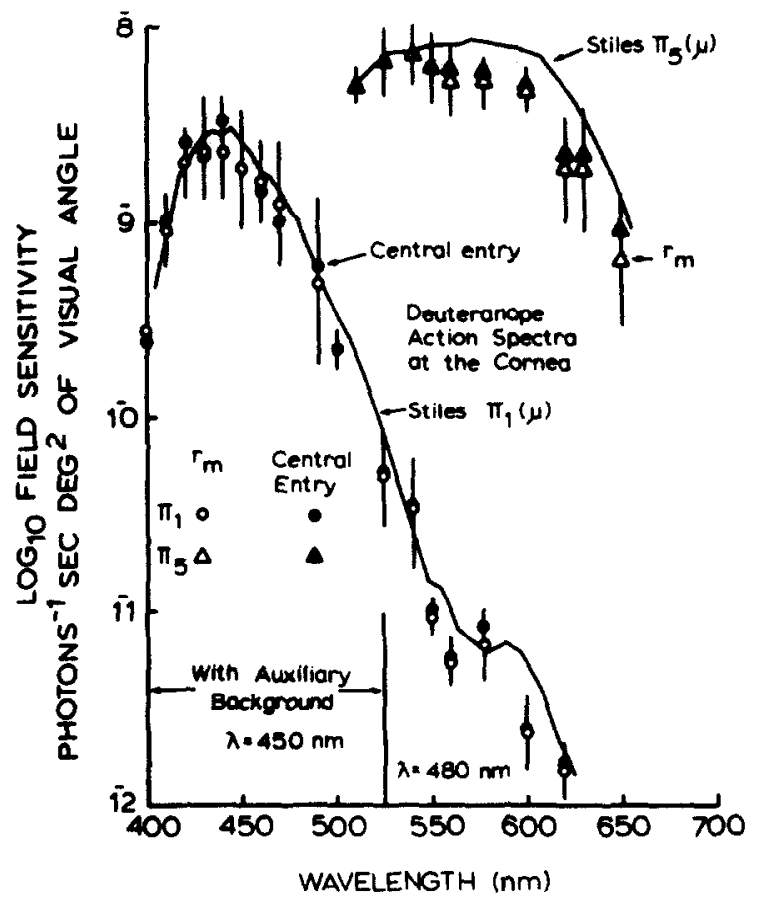

Fig. 4. The field sensitivity action spectra of $\pi_{1}(\mu)$ (circles) and $\pi_{5}(\mu)$ (triangles). The quantum flux density of the background which elevates the threshold of the mechanism by $1 \log$ unit above the absolute threshold is plotted as a function of wavelength. The solid symbols represent the action spectra for rays entering the pupil centrally in the preliminary experiments; the open symbols represent the action spectra at $r_{m}$ (the point of pupil entry of optimally effective rays as obtained by a computer search routine as the best estimate of the parabola parameters) in the main experiments. The curves are the field sensitivity $\pi_{1}(\mu)$ and $\pi_{5}(\mu)$ action spectra of Stiles (1959) plotted on this scale without shifting. ticularly for $\pi_{5}$ ) the correspondence is close enough to justify using this procedure to test the directional properties of these two mechanisms. This was done by repeating a series of experiments identical to those already described for background lights entering the eye every millimeter across the horizontal meridian of the pupil.

\section{B. Main experiments}

\section{No auxiliary background}

For each background wavelength a tvi curve was measured with background light entering every millimeter horizontally across the entrance pupil in a single half-day session with rest periods interspersed between determinations of each successive curve. A typical set of results obtained from one such session (with $550 \mathrm{~nm}$ background) is shown in Fig. 5. Each tvi curve displayed there, identified by number, was obtained for a different point of pupil entry. The template fit determined $S_{\mu}$, i.e. the $\log _{10}$ of the background intensity needed to elevate the threshold of $\pi_{j}$ $(j=1,5)$ by $1 \log$ unit, in every case. The two graphs in the lower middle of Fig. 5 show the plot of $S_{\mu}$ as a function of point of pupil entry $r$ (in $\mathrm{mm}$ )-below for $\pi_{1}(\mu)$, above for $\pi_{5}(\mu)$. A computer search routine was used to find the value of $a(\mu), r_{m}$ and $\rho(\mu)$ in the equation

$$
S_{\mu}=a(\mu)+\rho(\mu)\left(r-r_{m}\right)^{2}
$$

which provided the best fit to the empirical results, by minimizing the squares of the deviations. $a(\mu)$ defines the $\log$ of the field sensitivity action spectrum of the mechanism under study at $r_{m}$, the point of optimal pupil entry; $\rho(\mu)$ is the directional sensitivity of that mechanism to background fields. Stiles (1937) found that a similar equation describes the directional sensitivity of his own fovea; it is also a reasonable fit to the results for $\pi_{1}$ and $\pi_{5}$ obtained in this study, including those shown in Figs 5 and 6.

\section{Auxiliary backgrounds}

An analogous series with the auxiliary background for different points of pupillary entry is illustrated in Fig. 6 for a violet main background $(\mu=430 \mathrm{~nm})$.

The eight tvi curves so obtained are shown in this figure each identified by number ( 1 being $3 \mathrm{~mm}$ nasal, 8 being 

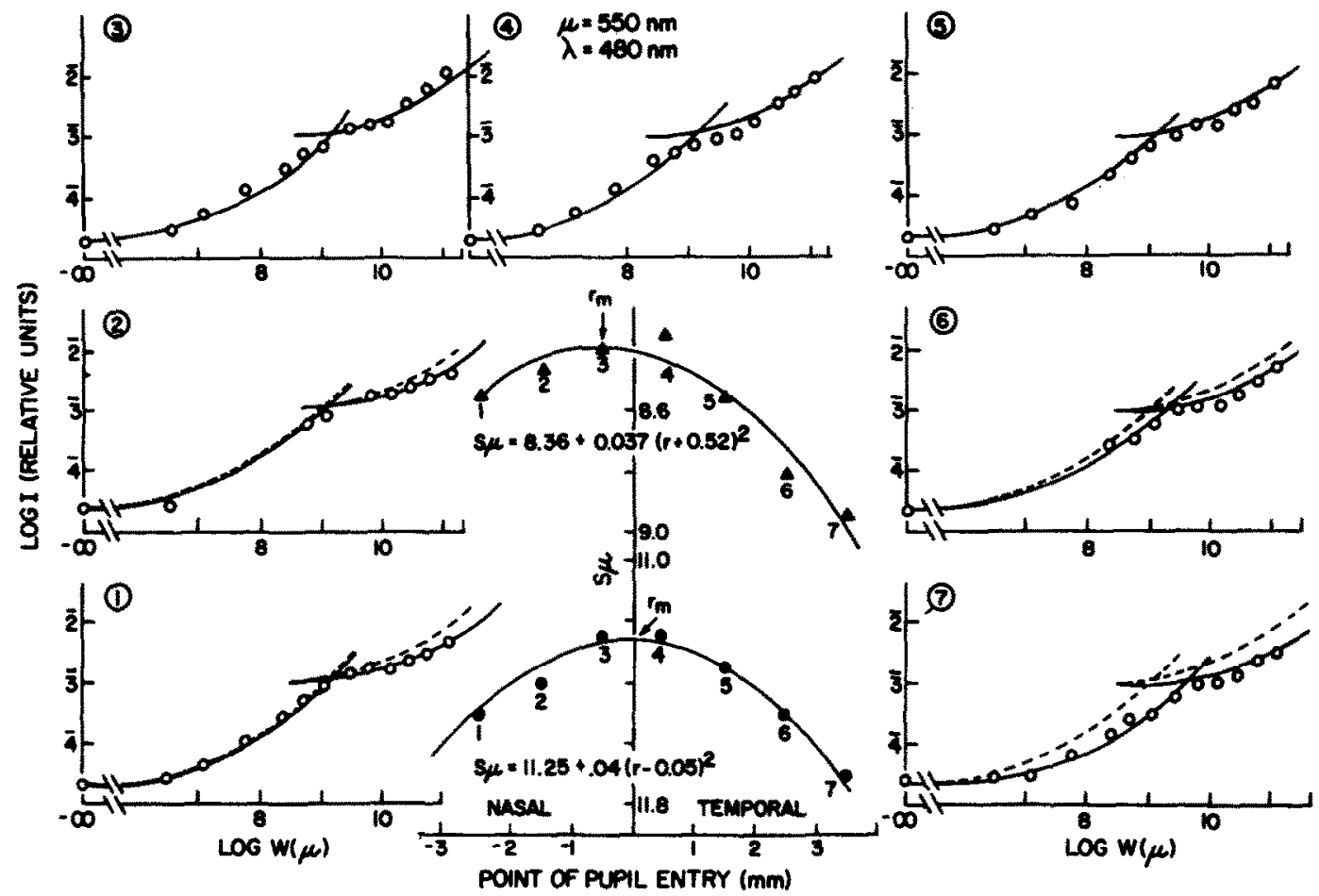

Fig. 5. Illustrated are seven tvi curves, each for a different point of pupil entry obtained on a single background wavelength $(550 \mathrm{~nm})$. The number on the graphs gives the point of entry in succession 1 ( $2.5 \mathrm{~mm}$ nasal) to 7 ( $3.5 \mathrm{~mm}$ temporal). The plotted points are the means of at least three experimental repetitions. Each tvi curve has two parts, a lower $\left(\pi_{s}\right)$ and an upper $\left(\pi_{1}\right)$ branch. The solid curve is the increment threshold $(\zeta)$ function of Stiles $(1953)$. For graphs which contain two sets of smooth curves, the left-hand set reproduces those obtained entering at the point of maximum pupil sensitivity. The lower middle graph is a plot of the field intensity, $S_{\mu}\left(\log _{10}\right.$ of background quanta $\left.\sec ^{-1} \mathrm{deg}^{-2}\right)$. raising the $\pi_{1}$ threshold 10-fold as a function of point of pupil entry. The middle graph represents a similar plot for $\pi_{5}(\mu)$. The smooth curve through each of these last two sets minimizes the square of the deviation from a parabola of the form defined by equation (1). Note that $r_{m}$ for $\pi_{1}(\mu)$ and $\pi_{5}(\mu)$ differ by $0.57 \mathrm{~mm}$.

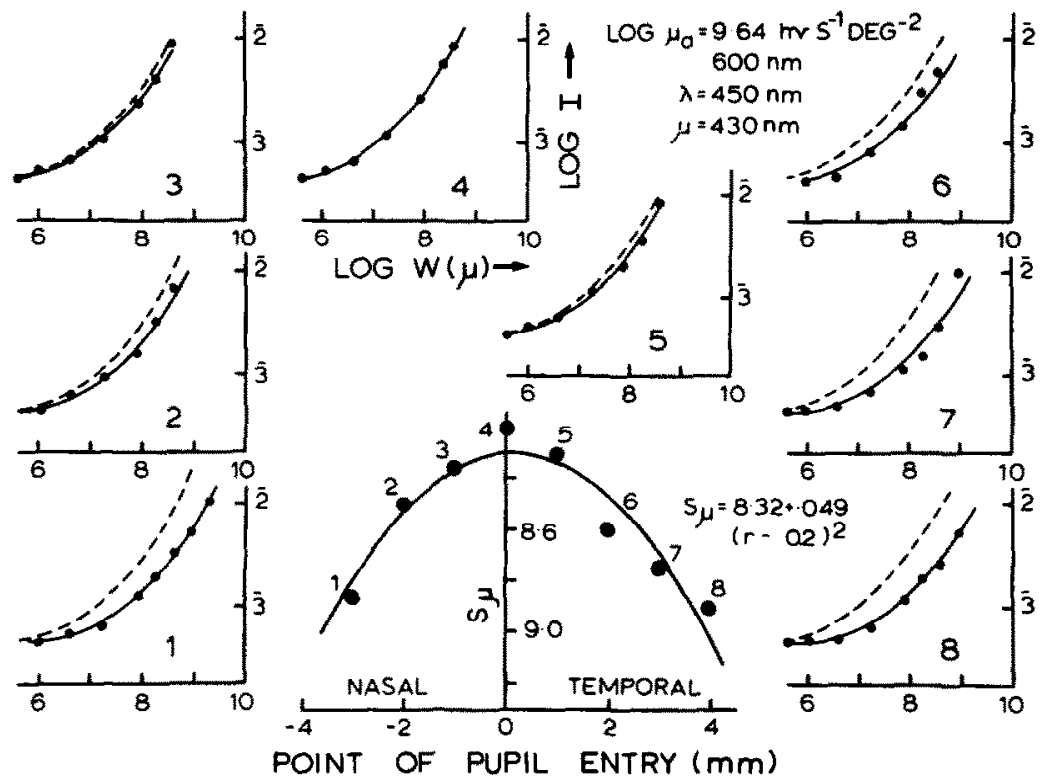

Fig. 6. Eight tvi curves are shown for main, test and auxiliary fields of $430 \mathrm{~nm}, 450 \mathrm{~nm}$ and $600 \mathrm{~nm}$, respectively. Each tvi curve represents the $\pi_{1}$ mechanism for a different point of entry of the main field background. The continuous curves are the Stiles increment threshold function $(\zeta)$. The left-hand curve is this curve for point 4 . The main field required to raise the threshold by one log unit (above its value on the auxiliary background) is represented by $S_{\mu}$ as a function of pupil position and is plotted below in the center. The continuous curve was fit to the data by a computer program which minimizes the squares of the deviations. 
$4 \mathrm{~mm}$ temporal, pupil entry). There is now no "change of law" and a single $\zeta$ template describes the experimental points. In each graph two such template curves are generally found: one is the eye fit to the experimental points the other (the left-hand-dotted - curve) is the eye fit to the measurements for $r=r_{m}$ (Graph 4). $S_{\mu}$, the log of the intensity required to raise the threshold by one $\log$ unit (above its values on the auxiliary background) was measured for each point of pupil entry and plotted in the center graph below. The computer search routine determined the values of $a(\mu), r_{m}$ and $\rho(\mu)$ of equation (1) which best described these data and the curve drawn through the points on this graph has the resulting equation. The fit is reasonable. A similar procedure was followed for all backgrounds equal to or less than $525 \mathrm{~nm}$.

\section{RESULTS}

The results of these experiments are most succinctly summarized by presenting the values of $a(\mu), \rho(\mu)$ and $r_{m}$ obtained from the computer search routine as the best estimates of the parabola parameters which described the measurements.

(i) $a(\mu)$. The values for $a(\mu)$ describe the action spectra of the two mechanisms at the optimum pupil entry point and since the conditions in this main experiment differ from those in the preliminary experiments only in that the latter apply to central pupil entry alone, they serve as a check on the repeatability and stability of this subject. The values $a(\mu)$ as a function of wavelength are plotted as open circles [for $\left.\pi_{1}(\mu)\right]$ and open triangles [for $\pi_{5}(\mu)$ ] in Fig. 4. The vertical lines enclose the geometric mean $\pm 1 \mathrm{SEM}$. There is good agreement between open and solid symbols attesting to the repeatability of these experimental measurements. The circles in this figure are also in accord with the spectrum defined by the left-hand continuous line, the (absolute) field sensitivity of $\pi_{1}(\mu)$ of Stiles. This is worthy of note considering individual differences in observer sensitivity, macular and lens pigmentation and subtle differences in techniques.

However, Stiles' estimates of $\pi_{5}(\mu)$ field sensitivity does not agree so well with those obtained here (triangles in Fig. 4). There are several possible explanations: Wald (1964) believes $\pi_{5}(\mu)$ is a composite of long-wave and middle-wave sensitive foveal cones. If so, $\pi_{5}$ in the deuteranopic retina (lacking the middle-wave sensitive cone visual pigment) would differ from that found in the normal retina. Unfortunately, the simplest pooling hypothesis would predict the triangle to be more, rather than less, sensitive in the middle wavelength $(540 \mathrm{~nm})$ than at longer wavelengths $(580 \mathrm{~nm})$. This is opposite to what is found (Fig. 4). However, there is no reason to expect the pooling to be simple and these results, therefore, do not exclude that possibility. Alternatively, the discrepancies may only be a reffection of individual differences in erythrolabe among different normals and different deuteranopes (Alpern and Wake, 1977; Alpern

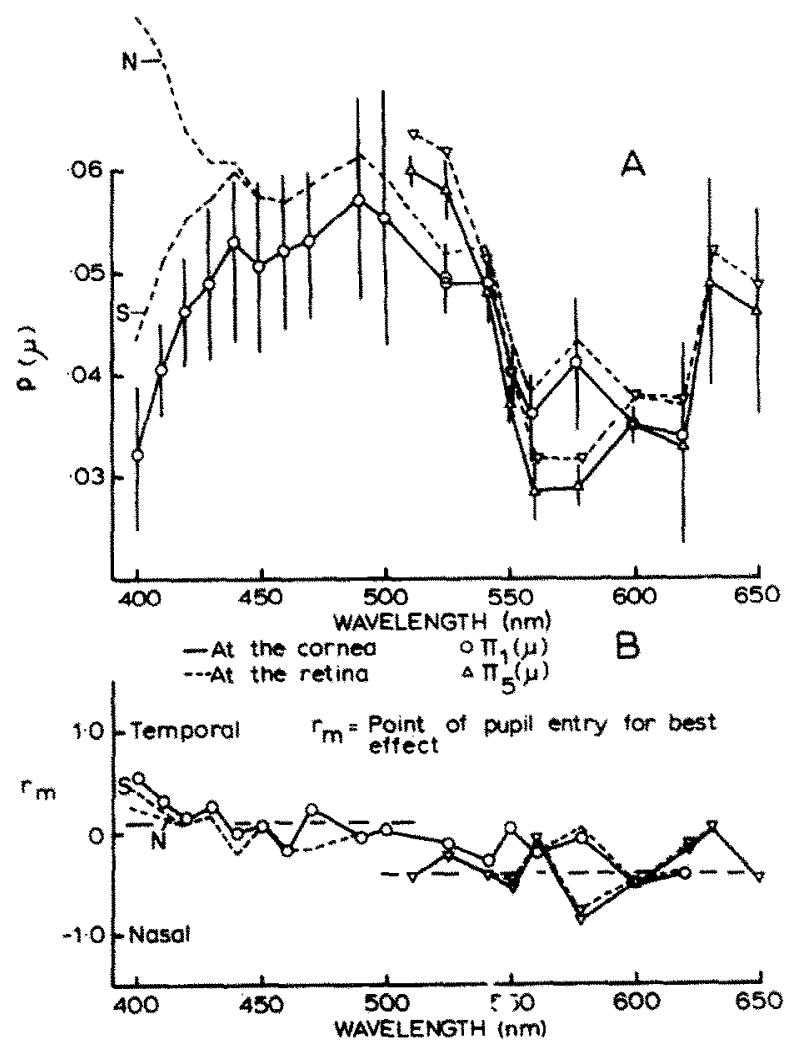

Fig. 7. The directional sensitivity $\rho(\mu)$ [A] and point of maximum pupil entry $r_{m}$ [B] plotted as functions of wavelength. The open circles represent $\pi_{1}(\mu)$, the triangles $\pi_{5}(\mu)$. The continuous lines connect the data points. The broken lines represent the results after correcting for losses in the eye media in a way described in the text. For $\mu<450 \mathrm{~nm}$ the corrections using Said-Weale's (S) estimate of the lens density differs from those in which the Norren-Vos $(\mathbf{N})$ table is employed. For $\mu \geqslant 450 \mathrm{~nm}$ the two estimates agree. 
and Pugh, 1977). These two are neither mutually exclusive, nor the only possibilities, and as yet nothing further has been done to sort out the most likely among them, since the measurements of $\pi_{5}$ were coincidental to the major thrust of the present paper.

(ii) $\rho(\mu)$. In Fig. 7A the open circles connected by the continuous line show the results for $\pi_{1}(\mu)$. The field sensitivity of this mechanism is optimally directionally sensitive at about $500 \mathrm{~nm}$, clearly less so either on the long, or the short, wave side. The triangles in this figure show the analogous results for $\pi_{5}(\mu)$. Evidently in the regions of the spectrum in which they overlap the relations between $\rho$ and $\mu$ for $\pi_{1}(\mu)$ and $\pi_{5}(\mu)$ have similar courses.

(iii) $r_{m}$. Finally, the best estimates of $r_{m}$ are plotted as continuous lines connecting circles (for $\pi_{1}$ ) and triangles (for $\pi_{5}$ ) each as a function of background wavelength in Fig. 7B. $\pi_{1}(\mu)$ shows a surprising wavelength dependent shift in $r_{m}$, not previously noted in the evaluation of the directional sensitivity of a single foveal mechanism. At the violet end of the spectrum $\pi_{1}$ is most sensitive to rays entering the pupil about $0.5 \mathrm{~mm}$ temporal to the pupil center but for backgrounds at wavelength of $600 \mathrm{~nm}, \pi_{1}$ is most sensitive to backgrounds which enter the pupil $0.5 \mathrm{~mm}$ nasal to its center. No similar trend is seen in $\pi_{5}(\mu)$ which within the (rather poor) precision of the estimate is most sensitive to rays entering $0.5 \mathrm{~mm}$ nasal.

\section{DISCUSSION}

The results, to be of use in theoretical treatments of the second kind of Stiles-Crawford effect, must be evaluated at the level of the retina after absorption (and to a less extent scattering) losses within the eye (principally in its lens). These losses, no doubt, vary both with wavelength of the light and with the region of the lens through which it must pass. Unfortunately, there is no way of knowing the precise values most appropriate for the $21 \mathrm{yr}$ old subject under study. The usual calculation makes the assumption that the losses arise from a single yellow substance uniformly distributed throughout the lens. Vos and van Os (1975) argue that this assumption is reasonable but their evidence is not compelling and the matter must remain open. However, in the absence of quantitative information which might provide a viable alternative, the corrections made here follow the usual practice. Estimates of the length of the light path through the lens for different points of pupil entry are available (Mellerio, 1971), but the greatest uncertainty relates to the absorbance spectrum of this yellow substance at any given point (say at the center) of entry to the lens. It is clear that this value varies with age; it is generally smaller and less yellow for young subjects. Estimates from such subjects in the literature are both direct and indirect. Most of the former come from enucleated eyes which are subject to the possibility of post mortem clouding in the hands of even the most careful investigators. The in vivo direct measurements of Alpern et al., (1965) do not extend for wavelengths below $420 \mathrm{~nm}$; only the direct measurements of Said and Weale (1959) are free of both these difficulties. Indirect estimates can be obtained, for example, by taking the difference between the action spectrum of night vision and the absorption spectrum of rhodop- sin (Norren and Vos, 1974). However, they are based upon assumptions (such as that the CIE scotopic visibility curve is derived from a single Dartnall monogram rhodopsin of $\lambda_{\max }$ density 0.2 at its $\lambda_{\max } 493 \mathrm{~nm}$ ) the validity of which the most recent microspectrophotometric results (Bowmaker et al., 1975; Bowmaker et al., 1977) offer strong reasons to doubt. Through most of the visible spectrum $\lambda \geqslant 450 \mathrm{~nm}$ the matter is only of academic interest because all in vivo estimates (direct and indirect) agree rather remarkably. Unfortunately, below $450 \mathrm{~nm}$ the indirect estimates infer a much higher density than Said and Weale's (1959) data suggest for people of the age of our subject (for example, the difference in density at $400 \mathrm{~nm}$ between Said and Weale's and the Norren-Vos estimates is more than $1.0 \log _{10}$ unit). As it turns out, this rather sharp difference introduces uncertainty in this correction in precisely the part of the spectrum where it is of theoretical importance, that is in the neighborhood of the $\lambda_{\max }$ of $\pi_{1}(\mu)$.

\section{Nature of $\pi_{1}(\mu)$}

The results in Fig. 7 have been corrected using the estimates of the density spectrum of the lens given both by Said and Weale and Norren and Vos (in conjunction with the path length estimate of Mellerio). The corrected value of each $S_{\mu}$ was used to recompute new best fitting parabolas. Then new values of $\rho(\mu)$ and $r_{m}$ are shown by the dotted lines in Figs 7A and 7B, respectively.

Directing attention to the latter results first it is evident that correcting for losses in the eye media does not significantly change the surprising wavelength dependency of $r_{m}$ for $\pi_{1}(\mu)$ noted above even though the extreme temporal value for $r_{m}$ at $400 \mathrm{~nm}$ is reduced slightly by the corrections (more so by using the Norren-Vos, than the Said-Weale, estimate).

A possible explanation for the residual (and still appreciable) wavelength dependency of $r_{m}$ in excitation of $\pi_{1}(\mu)$ is to be found in the suggestion that $\pi_{1}(\mu)$ may be a composite mechanism receiving input from more than one variety of foveal cones (Pugh, 1976; Augenstein and Pugh, 1977). In Pugh's model of $\pi_{1}(\mu)$ the test flash is detected by signals evoked by the absorption of light in short-wave sensitive cones after passing through two attenuators, the first being generated by the short-wave sensitive cones; in the normal the identity of the specics of cone (long- or middle-wave sensitive, or some combination of them) responsible for the second attenuation in Pugh's model is uncertain. This ambiguity is removed in the present instance since there can be only one other cone species in the deuteranopic fovea (Alpern and Wake, 1977) namely $\pi_{5}(\mu)$.

To account for the change in $r_{m}$ of $\pi_{1}(\mu)$ with wavelength it is further assumed that this subject's shortwave sensitive cones are pointed toward a part of the pupil (about $0.2 \mathrm{~mm}$ temporal to the center) slightly different from the part of the pupil to which his $\pi_{5}$ cones point $(0.5 \mathrm{~mm}$ nasal). The gradual change of $r_{m}$ with background wavelength change for $\pi_{1}(\mu)$ shown in Fig. 7B is about what would then be expected according to some pooling of the two cone mechanisms at intermediate wavelengths such as 
Pugh assumed to generate the field sensitivity action spectrum of $\pi_{1}(\mu)$. Alternatively the results in Fig. 7B could be described by a step transition at an intermediate wavelength between the short-wave sensitive cones and $\pi_{5}(\mu)$ though the values of $r_{m}$ assumed for the two cone systems would then be slightly different.

The idea that $\pi_{5}$ cones are pointed toward a different exit pupil point than short-wave sensitive cones is speculative, although a similar phenomenon has already been noted for short-wave sensitive cones of a blue cone monochromat (Alpern et al., 1971). No other way of dealing with the results in Fig. 7B is evident. Regardless of the validity of this speculation, the results in Fig. 7A provide good evidence that $\pi_{1}(\mu)$ is a composite of short-wave sensitive cones and $\pi_{5}$ cones, since in this figure the curves describing $\rho(\mu)$ for $\pi_{1}(\mu)$ and $\pi_{5}(\mu)$ run together within the margin of experimental error-in the part of the spectrum where they overlap. This can be appreciated by comparing the present measurements of $\rho(\mu)$ with those obtained by other methods. This is illustrated by the circles and squares (for $\pi_{1}$ ) and open triangles connected by the dotted lines $\left(\pi_{5}\right)$ in Fig. 8 which are a replot of the mean $\rho(\mu)$ results as a function of background wavelength from Fig. 7.

Plotted in this same figure is the directional sensitivity parameter $\rho$ of the short-wave sensitive ("blue") cones calculated from the color matching experiments of Enoch and Stiles (1961) (solid triangles) and the directional sensitivity $\rho(\lambda)$ obtained by Stiles (1939) testing the directional sensitivity of the test flash for $\pi_{1}(\lambda)$ (diamonds). The open circle shows the field sensitivity directionality at $580 \mathrm{~nm}$ measured for $\pi_{1}(\mu)$ and the $x$ that for $\pi_{4}(\mu)$ by Stiles (1939).
For wavelengths equal to, or smaller than, about $500 \mathrm{~nm}$ the three sets of results show remarkably similar wavelength dependent directional properties. Assuming that the observer of Stiles is slightly more (and that of Enoch and Stiles slightly less) sensitive than the present subject in this part of the spectrum, the three curves in Fig. 8 can be brought into coincidence by a slight vertical displacement [0.01 up, for Fnoch and Stiles: the same amount down for Stiles (1939)].

Elsewhere in the spectrum this is by no means the case. Directional sensitivity of $\pi_{1}(\lambda)$ (Stiles, 1939) sheds no light on such matters but precisely here, the calculations of Enoch and Stiles diverge sharply from both the present measurements and Stiles (1939) solitary test of the directional sensitivity of $\pi_{1}(\mu)$. Whether or not the calculation of Enoch and Stiles (1961) accurately estimates the wavelength properties of the short-wave sensitive cones, may be quite insensitive to this comparison if the directional properties of $\rho(\mu)_{\pi}$, for wavelengths longer than $500 \mathrm{~nm}$ reflect the directional properties of the deuteranope's $\pi_{5}$ foveal cones. The near coincidence of the $\rho(\mu)_{\pi_{s}}$ and $\rho(\mu)_{n}$, curves in Figs 6 and 7 in this part of the spectrum strongly support this interpretation.

Change in the action spectrum for $\pi_{1}(\mu)$ with change in the point of pupil entry

Returning to the way $\rho(\mu)$ varies with wavelength for $\pi_{1}(\mu)$, after allowing for losses in the lens (dotted line in Fig. 7A) the difficulty introduced by the discrepancy between the two estimates of lens absorption for light of wavelength $\mu \leqslant 450 \mathrm{~nm}$ is immediately evident. Beginning at $400 \mathrm{~nm}$, and moving to longer

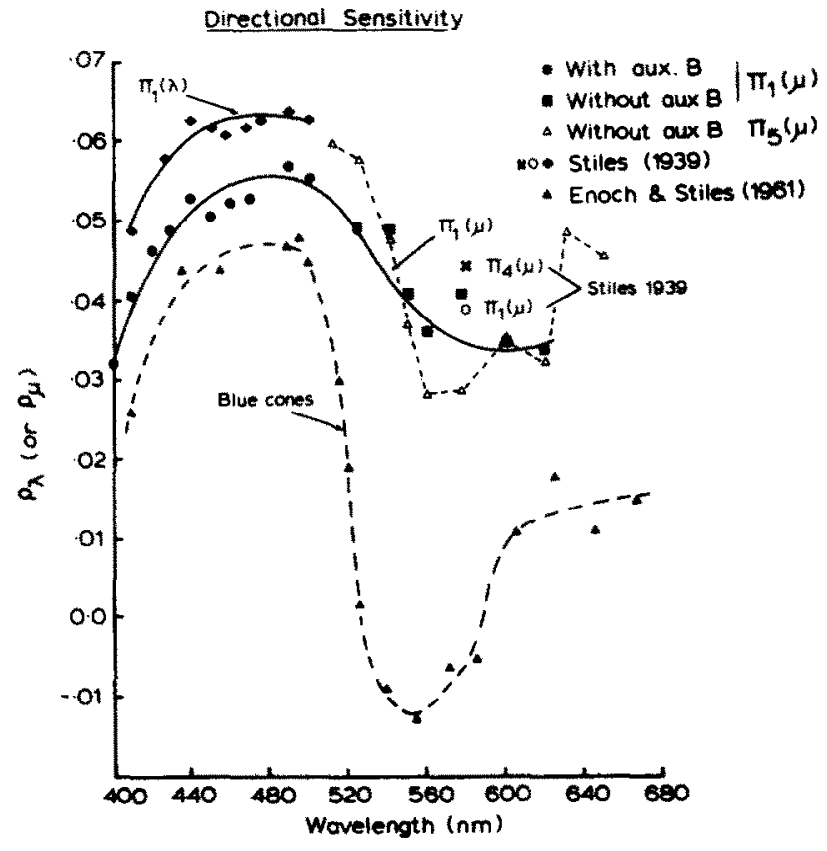

Fig. 8. Present measurements of $\rho(\mu)$ as a function of wavelength are compared with those obtained by other methods. The solid circles and squares represent the measured directional sensitivity of $\pi_{1}(\mu)$ with, and without, an auxiliary background, respectively. The open triangles represent the measurements of directional sensitivity for $\pi_{5}(\mu)$. The solid triangles represent the results of Enoch and Stiles (1961). The solid diamonds are the $\pi_{1}(\lambda)$ results obtained by Stiles (1939). The continuous and broken lines are drawn by hand to illustrate the trends. The open circle and the cross are values (for a $580 \mathrm{~nm}$ background) for $\pi_{1}(\mu)$ and $\pi_{4}(\mu)$, respectively, given by Stiles (1939, Fig. 43). 


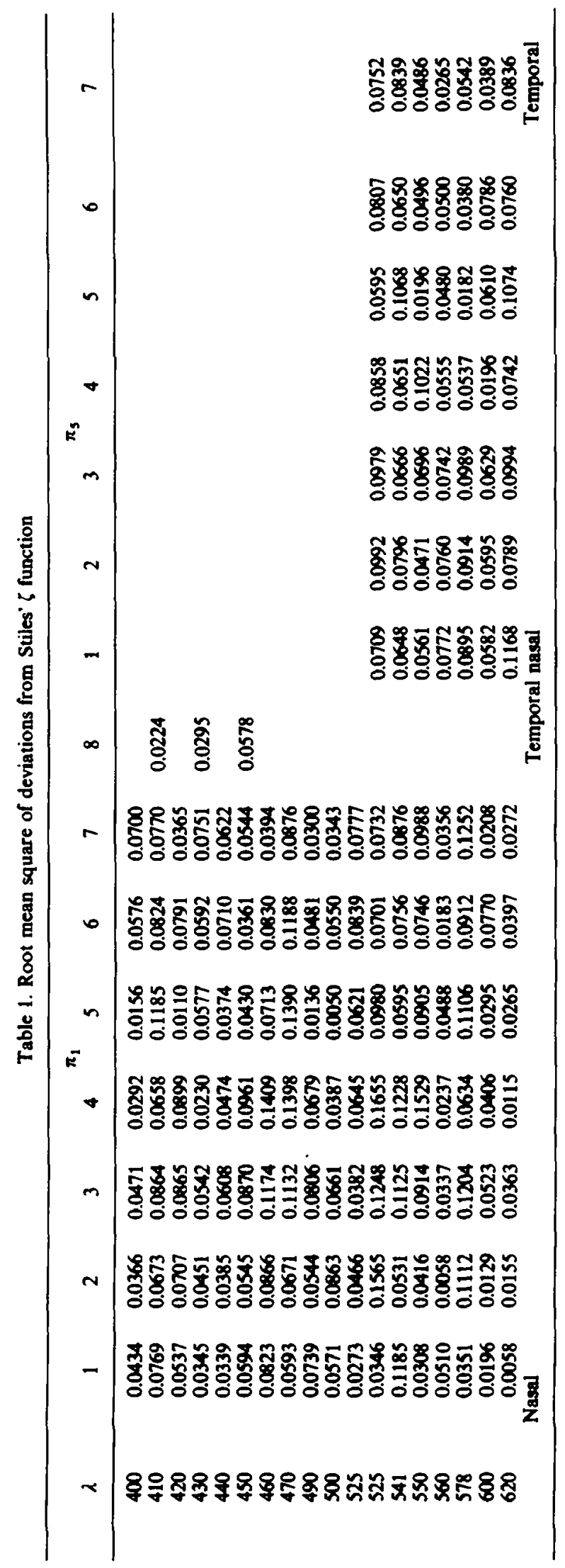




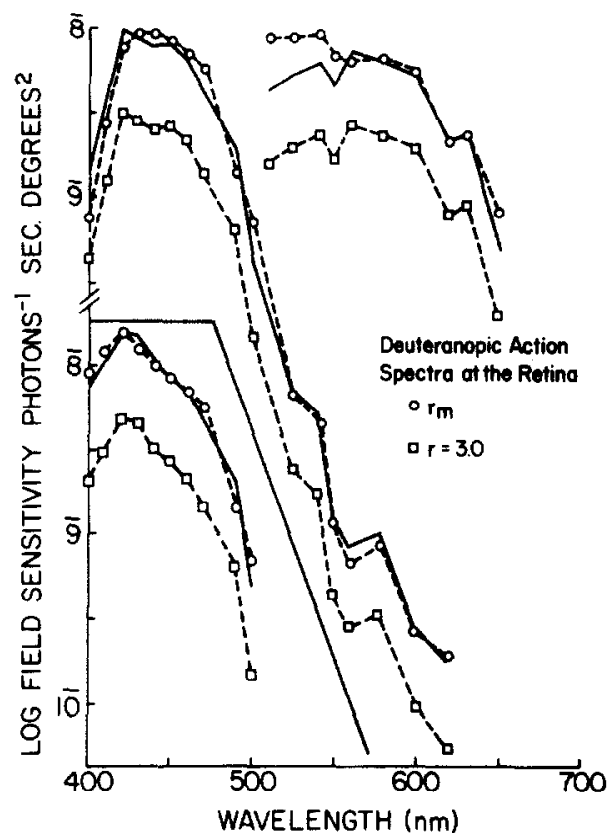

Fig. 9. The change in action spectra (corrected for eye media losses, as outlined in the text) of the two foveal cone mechanisms of the deuteranopic fovea on change of point of pupil entry from $r_{m}$ to a point $3.5 \mathrm{~mm}$ temporal to $r_{m}$. The upper pair of broken lines connecting the open circles represent the respective $\pi_{1}(\mu)$ and $\pi_{5}(\mu)$ action spectra for central (i.e. $r_{m}$ ) pupil entry. The dotted lines connecting the squares define the action spectra of these two mechanisms when the point of pupil entry has been shifted to $3.5 \mathrm{~mm}$ from $r_{m}$. The pair of continuous lines represents the respective action spectra for light entering the edge of the pupil $(r=3.5 \mathrm{~mm})$ shifted up so that near the peak they agree with the broken curves through the circles. The plot at lower left shows the results for $\mu \leqslant 500 \mathrm{~nm}$ when Norren-Vos estimate of lens density is used to calculate the correction. All other points have been corrected with the Said-Weale estimate.

wavelengths $\rho(\mu)$ gradually increases, assuming the conservative (Said-Weale) estimate. On the other hand, correcting according to Norren-Vos at $400 \mathrm{~nm}$ yields the largest value for $\rho(\mu)$ in this entire study (0.0746). The alternative "corrected" action spectra for light entering at $r_{m}$ and at $3.0 \mathrm{~mm}$ temporal to $r_{m}$ are illustrated in Fig. 9. In this figure the spectrum shown at the lower right is the result for $\pi_{1}(\mu)$ for wavelengths equal to or less than $500 \mathrm{~nm}$ correcting with the Norren-Vos table; all other points have been corrected with the Said-Weale estimate. A correction for absorption in the macular pigment (Wyszecki and Stiles, 1967) has also been introduced for all points plotted in Fig. 9. In the figure circles represent the corrected values for light entering through $r_{m}$, squares for that entering $3.0 \mathrm{~mm}$ temporal to $r_{m}$. To facilitate the comparison each continuous line is one member of the latter set shifted up so that at (or near) the wavelength of peak absorbance it coincides with the open circle for the corresponding member of the former set.

In keeping with the hypothesis of self-screening the spectral sensitivity of $\pi_{5}(\mu)$ for this subject is a maximum at very nearly the same wavelength that its directional sensitivity is a minimum. This is consistent with the conclusions Zwas (1979) obtained from other deuteranopes and Enoch and Stiles (1961) inferred for the long-wave sensitive cone mechanism from the measurements of the color of a light entering through the edge of the pupil (cf. also Brindley, 1953; Walraven and Bouman, 1960; Alpern, 1979).

In view of the uncertainty of the estimates of light losses in the lens at short wavelengths, however, it is impossible to be certain whether (or not) analogous considerations govern the directional properties of blue-sensitive cones. Depending only upon these corrections either possibility can be defended in the data given Pugh's theory of the composite nature of $\pi_{1}(\mu)$ which the present results strongly support. Whether or not a waveguide alternative such as the SnyderPask model (1973) would provide a better quantitative description also remains unresolved, for the identical reason.

In dealing with these issues the present study suggests that the best empirical approach might well be a systematic study of $\pi_{3}(\mu)$ on an observer about whom more explicit statements as to the losses of light within the lens are possible than is the case here.

Under the most favorable circumstances this is a task fraught with experimental difficulties (in part because of the high light intensities required) but perhaps not quite unsurmountable ones.

Even more formidable is the task of developing an explanation for the Enoch and Stiles (1961) prediction that the short-wave sensitive cones are more sensitive to monochromatic light of wavelengths in the neighborhood of $550 \mathrm{~nm}$ passing through the edge of the pupil than they are to that light when it passes through the center. This expectation was not experimentally confirmed here but the contradiction posed to it by Stiles' (1939) result was obviated by the strong suggestion that the directional sensitivity of $\pi_{1}(\mu)$ in this part of the spectrum was not due to the directional sensitivity of short-wave sensitive cones. A more direct confrontation may be expected if and when it becomes possible to measure the wavelength variation in the directional sensitivity of $\pi_{3}(\mu)$.

Acknowledgements-The work on this project was supported by Grant No. EY-00197 to M. Alpern from the National Eye Institute. F. Zwas was supported by an NEI postdoctoral fellowship (EY-505011), by a training grant for vision research to the University of Michigan EY-07022 and by a postdoctoral research fellowship ( $F-257)$ from Fight for Sight, Inc. (New York).

\section{REFERENCES}

Alpern M. (1968) Distal mechanisms of vertebrate colour vision. Ann. Rev. Physiol. 30, 279-318.

Alpern M. (1979) Lack of uniformity in colour matching. $J$. Physiol. 288, 85-105.

Alpern M., Lee G. B., Maaseidvaag F. and Miller S. S (1971) Colour vision in blue-cone "monochromacy". $J$. Physiol. 212, 211-233.

Alpern M. and Pugh E. N. Jr (1977) Variation in the action spectrum of erythrolabe among deuteranopes. J. Physiol. 266, 613-646.

Alpern M., Thompson S. and Lee M. S. (1965) Spectral transmittance of visible light by the living human eye. J. opt. Soc. Am. 55, 723-727.

Alpern M. and Wake T. (1977) Cone pigments in human deutan colour vision defects. J. Phsyiol. 266, 595-612. 
Augenstein E. J. and Pugh E. N. Jr (1977) The dynamics of the $\pi_{1}$ colour mechanism: further evidence for two sites of adaptation. J. Physiol. 272, 247-281.

Bowmaker J. K., Dartnall H. J. A., Lythgoe J. N. and Mollon J. D. (1978) The visual pigments of rods and cones in the Rhesus monkey Macaca mulatta. J. Physiol. 274, 329-348.

Bowmaker J. K., Loew E. R. and Liebman P. A. (1975) Variation in the $\lambda_{\max }$ of rhodopsin from individual rods. Vision Res. 15, 997-1003.

Boynton R. M., Ikeda M. and Stiles W. S. (1964) Interactions among chromatic mechanisms as inferred from positive and negative increment thresholds. Vision Res. 4, 87-117.

Brindley G. S. (1953) The effects on colour vision of adaptation to very bright lights. $J$. Physiol. 122, 332-350.

Cavonius C. R. and Estevez O. (1975) Contrast sensitivity of individual colour mechanisms of human vision. $J$. Physiol. 248, 649-662.

Du Croz J. J. and Rushton W. A. H. (1966) The separation of cone mechanisms in dark adaptation. J. Physiol. 183, 481-496.

Enoch J. M. and Stiles W. S. (1961) The colour changes of monochromatic light with retinal angle of incidence. Optica Acta 8, 329-358.

Estevez O. and Cavonius C. R. (1977) Human color perception and Stiles $\pi$ mechanisms. Vision Res. 17, 417-422.

Krauskopf J. and Mollon J. D. (1971) The independence of the temporal integration properties of individual chromatic mechanisms in the human eye. J. Physiol. 219, 611-623.

Mellerio J. (1971) Light absorption and scatter in the human lens. Vision Res. 11, 129-141.

Mollon J. D. and Polden P. G. (1977a) Saturation of retinal cone mechanisms. Nature 265, 243-246.

Mollon J. D. and Polden P. G. (1977b) An anomaly in the response of the eye to light of short wavelengths (with Appendix by Stiles W. S.). Phil. Trans. R. Soc. 278, 207-240.

Norren D. V. and Vos J. J. (1974) Spectral transmission of the human ocular media. Vision Res. 14, 1237-1244.

Pugh E. N. Jr (1976) The nature of $\pi_{1}$, colour mechanism of W. S. Stiles. J. Physiol. 257, 713-747.

Pugh E. N. Jr and Sigel C. (1978) Evaluation of the candidacy of the $\pi$-mechanisms of Stiles for color-matching fundamentals. Vision Res. 18, 317-330.

Rushton W. A. H. (1965) A foveal pigment in the deuteranope. J. Physiol. 176, 24-37.

Said F. S. and Weale R. A. (1959) The variation with age of the spectral transmissivity of the living human crystalline lens. Gerantologia 3, 213-231.

Sigel C. (1976) Field additivity in $\pi_{5}$ : some properties of the long-wavelength sensitive color mechanisms of $\mathrm{W}$. S. Stiles Ph.D. Thesis, University of Pennsylvania.

Snyder A. W. and Pask C. (1973) The Stiles-Crawford effect-explanation and consequences. Vision Res. 13, $1115-1137$.

Stiles W. S. (1937) The luminous efficiency of monochro- matic rays entering the eye pupil at different points and a new colour effect. Proc. R. Soc. B123, 90-118.

Stiles W. S. (1939) The directional sensitivity of the retina and the spectral sensitivities of the rods and cones. Proc. R. Soc. B127, 64-105.

Stiles W. S. (1946) Separation of the "blue" and "green" mechanisms of foveal vision by measurements of increment thresholds. Proc. R. Soc. B133, 418-434.

Stiles W. S. (1949) Increment thresholds and the mechanisms of colour vision. Documenta ophth. 3, 138-165.

Stiles W. S. (1953) Further studies of visual mechanisms by the two-colour threshold method. In Coloquio Sobre Problemas Opticas de la Vision. I. Conferencias Generales. Union Internationale de Physique Pure et Appliquée, pp. 65-103. Madrid

Stiles W. S. (1959) Color vision: The approach through increment-threshold sensitivity. Proc. natn. Acad. Sci. U.S.A. 45, 100-114.

Stiles W. S. (1967) Mechanism concepts in colour theory (Newton Lecture). J. Colour Group No. 11, 106-123.

Stiles W. S. (1978) Introductory Essay in Mechanisms of Colour Vision (edited by Stiles W. S.), pp. 1-34. Academic Press, London.

Vos J. J. and van Os F. L. (1975) The effect of lens density on the Stiles-Crawford effect. Vision Res. 15, 749-751.

Wald G. (1964) The receptors of human color vision. Science 145, 1007-1016.

Walraven P. L. and Bouman M. A. (1960) Relation between directional sensitivity and spectral response curves in human cone vision. J. opt. Soc. Am. 50, 780-784.

Wyszecki G. and Stiles W. S. (1967) Color Science. Concepts and Methods, Quantitative Data and Formulas. Wiley, New York.

Zwas F. (1979) Wavelength variation in directional sensjtivity of the long and medium wave sensitive foveal cones of red-green dichromats. Vision Res. This issue, p. 1067.

\section{APPENDIX}

Precision of the estimate of the fit of the

Stiles $\zeta$ template to individual tvi curves

In estimating the field sensitivities for the $\pi_{j}(j=1,5)$ mechanisms in this paper at each wavelength and each pupil entry point the Stiles $\zeta$ template was fit by eye to every tvi curve. The actual results are available on request to the authors. The root mean square of the deviations of the experimental points from this template fit is shown in every case in Table 1 . In this table columns numbered 1 through 8 represent points of pupil entry beginning at the nasal edge and progressing in $1 \mathrm{~mm}$ steps to the temporal edge in succession. The generally very small root mean square values found by fitting a previously established single curve of fixed shape by following the displacement rules of Stiles to all the experimental points of this study, testifies to the remarkable generality not only of the Stiles $\pi$ mechanism theory, but also to the accuracy of his empirically determined template. 